\title{
Low-cost alternatives to the Bethe-Salpeter equation: Towards simple hybrid functionals for excitonic effects in solids
}

\author{
Jiuyu Sun $\odot,{ }^{1}$ Jinlong Yang, ${ }^{2,3}$ and Carsten A. Ullrich $\oplus^{1, *}$ \\ ${ }^{1}$ Department of Physics and Astronomy, University of Missouri, Columbia, Missouri 65211, USA \\ ${ }^{2}$ Hefei National Laboratory for Physical Sciences at the Microscale, \\ University of Science and Technology of China, Hefei, Anhui 230026, China \\ ${ }^{3}$ Synergetic Innovation Center of Quantum Information \& Quantum Physics, \\ University of Science and Technology of China, Hefei, Anhui 230026, China
}

(Received 19 November 2019; published 28 January 2020)

\begin{abstract}
The Bethe-Salpeter equation (BSE) is the standard computational method for optical excitations in solids, including excitonic effects. In this paper we explore ways to reduce the computational cost of the BSE by simplifying the dielectrically screened Coulomb interaction: Instead of calculating the dielectric function from first principles, we replace it by a momentum-dependent model dielectric function or just by a single parameter. Combined with a semilocal exchange-correlation kernel, this defines an alternative class of hybrid functionals for solids within generalized time-dependent density-functional theory. We perform a systematic assessment of these simplified approaches and find that they yield optical absorption spectra and exciton binding energies of semiconductors and wide-gap insulators in close agreement with the standard BSE and with experiment. We also present applications to the perovskite material $\mathrm{CsPbBr}_{3}$ as an example of a more complex system.
\end{abstract}

DOI: 10.1103/PhysRevResearch.2.013091

\section{INTRODUCTION}

Excitonic effects play a critical role in the optical properties of electronic materials used in light-emitting devices, photovoltaics, and photocatalysts. The standard method to describe the excitons-coupled and correlated electron-hole quasiparticles (QPs) - is through a Green's-function-based approach known as the Bethe-Salpeter equation (BSE) [1-3]. For extended systems, the BSE is the most accurate method to calculate optical properties [4-6], but this accuracy tends to come with a rather high computational cost.

Excitons originate from the Coulomb interactions within the electron-hole pairs that are created during optical excitation processes. Coulomb interactions in electronic materials have classical and nonclassical contributions, also known as direct (or Hartree) and exchange-correlation (xc); for excitons, screened Coulomb exchange (where the screening can be viewed as a form of correlation) is the key mechanism of electron-hole interaction, whereas the Hartree interaction gives rise to so-called local-field effects, which are less influential. The BSE accounts for all of these effects from first principles.

The main computational effort within the BSE is spent on the screened Coulomb exchange interactions, which require

\footnotetext{
*ullrichc@missouri.edu

Published by the American Physical Society under the terms of the Creative Commons Attribution 4.0 International license. Further distribution of this work must maintain attribution to the author(s) and the published article's title, journal citation, and DOI.
}

constructing the inverse dielectric function using the randomphase approximation (RPA) [7-9]. Although dynamical effects are usually neglected, it is still costly to perform RPA calculations to get fully converged dielectric functions $\varepsilon^{\mathrm{RPA}}$, since many unoccupied bands and a relatively dense $\mathbf{k}$-grid sampling are typically needed.

Over the past decades, remarkable progress has been made in simplifying the RPA calculations or developing model dielectric functions [10-13]. By substituting $\varepsilon^{\mathrm{RPA}}$ with such model dielectric functions, not only the optical properties but also the quasiparticle band structure (within the $G W$ approximation) can be reasonably estimated for a wide range of materials [11,14-17].

An alternative approach to the optical excitation problem is time-dependent density-functional theory (TDDFT) [18-20]. Using xc kernels with a proper long range, TDDFT can successfully describe excitonic effects and produce reasonable optical spectra for solids [21-28].

A very widely used generalization of (TD)DFT [29-31] is based on hybrid functionals, where a fraction of nonlocal (Hartree-Fock) exchange is combined with semilocal exchange and correlation [32-36]. In ground-state DFT, hybrid $\mathrm{xc}$ functionals have gained increased popularity for calculating electronic band structures, since they offer a practical solution to DFT's band-gap problem [37-49]. There also are a few applications of hybrid xc functionals to describe excitations in periodic solids [50-53]. In particular, the so-called optimally tuned range-separated hybrids produce excellent results for organic molecular crystals [54,55]. Recently, Wing and co-workers $[56,57]$ showed that range-separated hybrids with an empirical parameter agree well with $G W$ BSE results for several materials. 
In a similar context, a screened exact exchange (SXX) approach was recently proposed by Yang et al. [58]. The SXX can be viewed as a simplified BSE approach, where the dielectric function is replaced by a uniform screening parameter $\gamma$, but it can also be seen as a special type of hybrid functional where the semilocal xc part is not included. In the original work [58], $\gamma$ was determined nonempirically as the inverse of the dielectric constant. This approximation only requires RPA calculations at $\mathbf{q}=0$, leading to a significant computational speedup. Moreover, $\gamma$ can also be obtained using dielectric constants from experiment.

The purpose of this paper is a systematic assessment of simplified BSE schemes, including BSE based on model dielectric functions, SXX, and a proposed hybrid functional which combines SXX with a fraction of local exchange and correlation. The primary goal is to find a nonempirical approach which can be used to calculate excitonic effects in complex materials for which the standard BSE is unaffordable, but with similar (or perhaps even better) accuracy compared to experiment.

We will mainly focus on calculating exciton binding energies and optical spectra in simple semiconducting and insulating materials, with particular emphasis on practical aspects such as convergence with respect to the number of reciprocal lattice vectors, and robustness under small variations of the dielectric screening. To demonstrate the capability of the simplified BSE schemes, we also present a detailed study of the cubic perovskite material $\mathrm{CsPbBr}_{3}$. The performance of the simplified BSE schemes turns out to be excellent throughout; the hybrid functional is found to work particularly well for the wide-gap insulators LiF and solid Ar.

The paper is organized as follows. In Sec. II we present the theoretical background of the BSE formalism and its simplifications and discuss some computational details. In Sec. III we present results for a variety of common insulators and semiconductors, as well as for $\mathrm{CsPbBr}_{3}$, and we assess the performance of the simplified BSE schemes. Section IV contains our conclusions and a general outlook. Various additional technical details are given in the Appendix.

\section{THEORY AND METHODOLOGY}

\section{A. Simplifying the dielectric screening in the BSE}

The BSE can be expressed as a matrix equation [59]

$$
\left(\begin{array}{cc}
\mathbf{A} & \mathbf{B} \\
\mathbf{B}^{*} & \mathbf{A}^{*}
\end{array}\right)\left(\begin{array}{l}
\mathbf{X}_{n} \\
\mathbf{Y}_{n}
\end{array}\right)=\omega_{n}\left(\begin{array}{cc}
-\mathbf{1} & \mathbf{0} \\
\mathbf{0} & \mathbf{1}
\end{array}\right)\left(\begin{array}{l}
\mathbf{X}_{n} \\
\mathbf{Y}_{n}
\end{array}\right),
$$

where $\mathbf{A}$ and $\mathbf{B}$ are defined in quasiparticle transition space (including excitations and deexcitations), $\left(\mathbf{X}_{n}, \mathbf{Y}_{n}\right)$ are the $n$th eigenvectors, and $\omega_{n}$ is the $n$th excitation energy. The deexcitation effects are usually negligible in the BSE with momentum transfer $\mathbf{q}=0$ [28,60]. Therefore, we here adopt the Tamm-Dancoff approximation, which means $\mathbf{B}$ is set to zero [27,60]. Then Eq. (1) can be simplified as

$$
\left[\left(E_{c, \mathbf{k}}-E_{v, \mathbf{k}^{\prime}}\right) \delta_{v v^{\prime}} \delta_{c c^{\prime}} \delta_{\mathbf{k} \mathbf{k}^{\prime}}+K_{c v \mathbf{k}, c^{\prime} v^{\prime} \mathbf{k}^{\prime}}^{\mathrm{BSE}}\right] \mathbf{Y}_{n}=\omega_{n} \mathbf{Y}_{n} .
$$

Here $v$ denotes occupied valence bands, $c$ denotes unoccupied conduction bands, and the $E_{\mathbf{k}}$ are single-(quasi)particle energies. Equation (2) features the coupling matrix $K^{\mathrm{BSE}}=$ $K^{\mathrm{d}}+K^{\mathrm{x}}$, which is also called the BSE kernel. The first part of $K^{\mathrm{BSE}}$ is the direct interaction

$$
K^{\mathrm{d}}=\frac{2}{V_{\text {cell }}} \sum_{\mathbf{G} \neq 0} \frac{4 \pi}{|\mathbf{G}|^{2}}\left\langle c \mathbf{k}\left|e^{i \mathbf{G} \cdot \mathbf{r}}\right| v \mathbf{k}\right\rangle\left\langle v^{\prime} \mathbf{k}^{\prime}\left|e^{-i \mathbf{G} \cdot \mathbf{r}}\right| c^{\prime} \mathbf{k}^{\prime}\right\rangle,
$$

which will remain the same for all the methods in the present work. The second part is the exchange kernel

$$
\begin{aligned}
K^{\mathrm{x}}(\mathbf{q}, \omega)= & \frac{2}{V_{\text {cell }}} \sum_{\mathbf{G}, \mathbf{G}^{\prime}} W_{\mathbf{G}, \mathbf{G}^{\prime}}(\mathbf{q}, \omega) \delta_{\mathbf{q}, \mathbf{k}-\mathbf{k}^{\prime}} \\
& \times\left\langle c \mathbf{k}\left|e^{i(\mathbf{q}+\mathbf{G}) \mathbf{r}}\right| c^{\prime} \mathbf{k}^{\prime}\right\rangle\left\langle v^{\prime} \mathbf{k}^{\prime}\left|e^{-i\left(\mathbf{q}+\mathbf{G}^{\prime}\right) \mathbf{r}}\right| v \mathbf{k}\right\rangle,
\end{aligned}
$$

which is much more expensive to calculate than $K^{\mathrm{d}}$. The main computational cost comes from the construction of the screened Coulomb interaction $W_{\mathbf{G}, \mathbf{G}^{\prime}}(\mathbf{q})$, given by

$$
W_{\mathbf{G}, \mathbf{G}^{\prime}}(\mathbf{q}, \omega)=-\frac{4 \pi \varepsilon_{\mathbf{G}, \mathbf{G}^{\prime}}^{-1}(\mathbf{q}, \omega)}{|\mathbf{q}+\mathbf{G}|\left|\mathbf{q}+\mathbf{G}^{\prime}\right|} .
$$

The inverse dielectric function $\varepsilon_{\mathbf{G}, \mathbf{G}^{\prime}}^{-1}(\mathbf{q}, \omega)$ is obtained from first principles via

$$
\varepsilon_{\mathbf{G}, \mathbf{G}^{\prime}}^{-1}(\mathbf{q}, \omega)=\delta_{\mathbf{G}, \mathbf{G}^{\prime}}+\frac{4 \pi}{|\mathbf{q}+\mathbf{G}|^{2}} \chi_{\mathbf{G}, \mathbf{G}^{\prime}}^{\mathrm{RPA}}(\mathbf{q}, \omega),
$$

where $\chi_{\mathbf{G}, \mathbf{G}^{\prime}}^{\mathrm{RPA}}(\mathbf{q}, \omega)$ is the density-density response function in the random-phase approximation.

For most practical BSE calculations, a widely used treatment is to ignore the frequency dependence of the dielectric function and use $\varepsilon_{\mathbf{G}, \mathbf{G}^{\prime}}^{-1}(\mathbf{q}, \omega=0)[4,5,61]$. However, the $\omega$ dependent dynamic effects of the dielectric screening may have a non-negligible influence on the electron dynamics $[62,63]$, which can be estimated by methods such as generalized plasmon-pole models [64-66]. In the present work we use the static approximation throughout and refer to this as the standard BSE approach or simply as the BSE.

Even if the static approximation is made, the RPA calculation for the dielectric function is still one of the most resourcedemanding steps for the whole process of first-principles BSE calculations, because it requires a summation over the $\mathbf{q}$ grid in the reduced Brillouin zone, in addition to a double sum over the reciprocal wave vectors $\mathbf{G}$ and $\mathbf{G}^{\prime}$. Furthermore, many unoccupied bands must be included in $\chi^{\text {RPA }}$ to obtain a fully converged dielectric function.

Alternatively, one can replace the RPA dielectric function by a model dielectric function or just a simple parameter, both of them diagonal in $\mathbf{G}, \mathbf{G}^{\prime}$ :

$$
\varepsilon_{\mathbf{G}, \mathbf{G}^{\prime}}^{-1}(\mathbf{q}, 0) \longrightarrow \begin{cases}\varepsilon(q)^{-1} \delta_{\mathbf{G}, \mathbf{G}^{\prime}} & (\mathrm{m}-\mathrm{BSE}) \\ \gamma \delta_{\mathbf{G}, \mathbf{G}^{\prime}} & (\mathrm{SXX}) .\end{cases}
$$

Here m-BSE stands for the BSE with a model dielectric function and SXX stands for screened exact exchange, following Ref. [58] (if $\gamma=1$, the method reduces to time-dependent Hartree-Fock, hence the name SXX). We also considered the head-only SXX (h-SXX), where we set $\varepsilon_{\mathbf{G}, \mathbf{G}^{\prime}}^{-1}(\mathbf{q}, 0)=$ $\gamma \delta_{\mathbf{G}, \mathbf{G}^{\prime}} \delta_{\mathbf{G}, 0}$.

Here we adopt the model dielectric function by Cappellini et al. [13],

$$
\varepsilon(q)=1+\left\{\frac{1}{\varepsilon(0)-1}+\alpha\left(\frac{q}{q_{\mathrm{TF}}}\right)^{2}+\frac{\hbar^{2} q^{4}}{4 m^{2} \omega_{p}^{2}}\right\}^{-1},
$$


where $q=|\mathbf{q}+\mathbf{G}|, \alpha=1.563$ is an empirical parameter, and $q_{\mathrm{TF}}$ and $\omega_{p}$ are the Thomas-Fermi wave vector and the plasma frequency, respectively, obtained with the average electron density of the system. Finally, $\varepsilon(q)$ requires the dielectric constant $\varepsilon(0)$ as input, which can be either taken from experiment or calculated as the head of the RPA inverse dielectric function $\varepsilon(0)=1 /\left(\varepsilon^{\mathrm{RPA}}\right)_{0,0}^{-1}(\mathbf{q}=0)$, using Eq. (6).

In Ref. [58] it was proposed that the screening parameter $\gamma$ in SXX be determined from a first-principles RPA calculation:

$$
\gamma=\left(\varepsilon^{\mathrm{RPA}}\right)_{0,0}^{-1}(\mathbf{q}=0)=1 / \varepsilon(0) .
$$

In fact, the role of $\varepsilon_{0,0}^{-1}$ could be regarded as an overall account of the Coulomb screening effects for the optical excitation when $\mathbf{q} \rightarrow 0$. Furthermore, the $\varepsilon(0)$ or $\gamma$ in the m-BSE and SXX can also be treated as empirically adjustable parameters. Such flexibility could be helpful when the RPA dielectric function alone is not sufficiently accurate for excitonic effects, for instance, due to the presence of lattice screening effects [67]. We use the definition of the screening parameter in Eq. (9) for m-BSE, SXX, and the hybrid functional kernel throughout, unless explicitly stated otherwise.

The main advantage of m-BSE and SXX is avoiding the full RPA calculation of the momentum-dependent dielectric function. Another advantage is that the off-diagonal terms of $W_{\mathbf{G}, \mathbf{G}^{\prime}}$ are eliminated. As we will see below, the off-diagonal terms of the dielectric function are not important for the optical response in semiconductors, but in insulators with strong short-range interactions, the effect of the off-diagonal terms [local-field effects (LFEs)] can be non-negligible. Overall, it will turn out that the simplified schemes developed here agree closely with the BSE while leading to a significant reduction of computational cost (as detailed in Appendix D).

\section{B. From the simplified BSE to generalized TDDFT: A hybrid functional for excitons in solids}

In ground-state DFT, the central idea of hybrid functionals is to write the xc energy functional as [32-36]

$$
E_{\mathrm{xc}}^{\mathrm{hybrid}}=a E_{\mathrm{x}}^{\mathrm{exact}}+(1-a) E_{\mathrm{x}}^{\mathrm{sl}}+E_{\mathrm{c}}^{\mathrm{sl}},
$$

where $E_{\mathrm{x}}^{\text {exact }}$ is the exact Fock exchange energy functional and $E_{\mathrm{x}, \mathrm{c}}^{\mathrm{sl}}$ are approximate semilocal exchange and correlation functionals, respectively. The parameter $a$ (often chosen as $a \approx 0.25$ ) mixes exact exchange with semilocal exchange.

While hybrid functionals have found widespread application for periodic solids due mainly to their excellent performance for calculating band gaps [37-49], there have so far been relatively few applications of hybrid functionals for optical spectra in semiconductors and insulators [50-57]. Here we propose a nonempirical hybrid approach specifically designed to produce excitonic properties in close agreement with the BSE. The idea is to combine the long-range SXX with an approximate treatment of xc local-field effects via the adiabatic LDA (ALDA), in the following way: In the BSE equation (2) we replace the screened exchange part of the BSE kernel $K^{\mathrm{x}}$ with the hybrid kernel

$$
K_{\mathrm{xc}}^{\mathrm{hybrid}}=K^{\mathrm{SXX}}+(1-\gamma) K_{\mathrm{xc}}^{\mathrm{ALDA}},
$$

where

$$
\begin{aligned}
K^{\mathrm{SXX}}(\mathbf{q})= & -\frac{2}{V_{\text {cell }}} \sum_{\mathbf{G}} \delta_{\mathbf{q}, \mathbf{k}-\mathbf{k}^{\prime}} \frac{4 \pi \gamma}{|\mathbf{q}+\mathbf{G}|^{2}} \\
& \times\left\langle c \mathbf{k}\left|e^{i(\mathbf{q}+\mathbf{G}) \mathbf{r}}\right| c^{\prime} \mathbf{k}^{\prime}\right\rangle\left\langle v^{\prime} \mathbf{k}^{\prime}\left|e^{-i(\mathbf{q}+\mathbf{G}) \mathbf{r}}\right| v \mathbf{k}\right\rangle
\end{aligned}
$$

and

$$
\begin{aligned}
K_{\mathrm{xc}}^{\mathrm{ALDA}}(\mathbf{q})= & \frac{2}{V_{\text {cell }}} \lim _{\mathbf{q} \rightarrow 0} \sum_{\mathbf{G}, \mathbf{G}^{\prime}} f_{\mathrm{xc}, \mathbf{G} \mathbf{G}^{\prime}}^{\mathrm{ALDA}}(\mathbf{q}) \\
& \times\left\langle c \mathbf{k}\left|e^{i(\mathbf{q}+\mathbf{G}) \mathbf{r}}\right| v \mathbf{k}\right\rangle\left\langle v^{\prime} \mathbf{k}^{\prime}\left|e^{-i\left(\mathbf{q}+\mathbf{G}^{\prime}\right) \mathbf{r}}\right| c^{\prime} \mathbf{k}^{\prime}\right\rangle .
\end{aligned}
$$

Here $f_{\mathrm{xc}, \mathbf{G G}}^{\mathrm{ALDA}}(\mathbf{q})$ is the local frequency-independent xc kernel in the ALDA [20].

The construction of our hybrid kernel (11) differs from standard generalized (TD)DFT hybrid expressions [see Eq. (10)] in that we reduce the entire local xc part by a factor $1-\gamma$, whereas in $E_{\mathrm{xc}}^{\mathrm{hybrid}}$ only the exchange part is reduced and not the correlation part. The reason for this is that the screening in SXX is in essence a correlation effect, so we must reduce all of $K_{\mathrm{xc}}^{\mathrm{ALDA}}$ (not just the exchange part) in order to avoid overcounting of correlation. While this is of course just a simple approximation, it nevertheless captures essential aspects of the behavior of screened electron dynamics in solids and, as we will see below, $K_{\mathrm{xc}}^{\text {hybrid }}$ leads to good results.

\section{Computational details}

To calculate Kohn-Sham band structures, we used the LDA functional in the QUANTUM ESPRESSO package [68], employing a plane-wave basis along with optimized norm-conserving Vanderbilt pseudopotentials [69]. The electronic band gaps were then corrected to the experimental value by applying scissors operators [70,71]. Experimental lattice parameters were used for all materials.

With the so-obtained Kohn-Sham band structures, we used the YAMBO code [72] to perform our calculations for optical excitations. We used a $28 \times 28 \times 28 \Gamma$-centered k-point mesh for $\mathrm{GaAs}$ and $\mathrm{Si}$. The $\mathbf{k}$ grids for the other materials are $24 \times$ $24 \times 24$ for $\mathrm{z}-\mathrm{GaN}$ and $\mathrm{CdS}, 24 \times 24 \times 12$ for $\mathrm{w}-\mathrm{GaN}$ and AlN, and $16 \times 16 \times 16$ for LiF and Ar. The RPA dielectric functions were calculated with at least 60 conduction bands and $200 \mathbf{G}$ vectors. To build the BSE and ALDA kernels, we used three valence bands and three conduction bands for GaAs and Ar. The corresponding numbers of valence and conduction bands for the other materials are four valence and four conduction bands for z-GaN, Si, w-GaN, and AlN and five valence and five conduction bands for $\mathrm{CdS}$ and $\mathrm{LiF}$ (with the $1 s$ electrons of Li included in the pseudopotential).

We used Haydock iteration to solve the BSE-type equations, instead of directly diagonalizing the huge BSE matrix. To identify the position of excitons in the spectra, a smaller broadening of $0.002 \mathrm{eV}$ is used; to calculate optical spectra, a much larger broadening of $0.1 \mathrm{eV}$ is used. It is well known that large numbers of $\mathbf{k}$ points are required to obtain converged spectra $[73,74]$. We adopted the random integration method and inversion solver in YAMBO [72,75]. In this scheme, the optical spectra of $\mathrm{z}-\mathrm{GaN}$ are calculated with a double $\mathbf{k}$ grid, which includes a $12 \times 12 \times 12 \Gamma$-centered uniform k-point mesh and 20000 random interpolated $\mathbf{k}$ points. 
TABLE I. Exciton binding energies for various elemental and binary materials, comparing calculated and experimental results. All energies are in $\mathrm{meV}$, except for $\mathrm{LiF}$ and $\mathrm{Ar}(\mathrm{eV})$. The experimental data are from Refs. [76-86].

\begin{tabular}{lcccccccc}
\hline \hline Approach & GaAs & Si & CdS & z-GaN & w-GaN & AlN & LiF & Ar \\
\hline BSE & 24 & 42 & 59 & 103 & 110 & 181 & 2.05 & 1.83 \\
m-BSE & 24 & 43 & 59 & 103 & 108 & 185 & 2.17 & 1.78 \\
SXX & 24 & 41 & 58 & 101 & 106 & 177 & 1.93 & 1.75 \\
h-SXX & 25 & 43 & 58 & 101 & 106 & 176 & 1.77 & 1.70 \\
Hybrid & 24 & 41 & 59 & 102 & 107 & 180 & 2.00 & 1.80 \\
Expt. & 4 & 14 & 28 & 26 & 20 & 75 & 1.6 & 1.9 \\
\hline \hline
\end{tabular}

\section{RESULTS AND DISCUSSION}

\section{A. Elemental and binary materials}

We assess the performance of m-BSE, SXX, and the hybrid kernel for $\mathrm{Si}$ and for several common binary compounds, namely, the semiconductors $\mathrm{GaAs}, \mathrm{GaN}$ with zinc-blende and wurtzite structure ( $\mathrm{z}-\mathrm{GaN}$ and $\mathrm{w}-\mathrm{GaN}$, respectively), AlN, and $\mathrm{CdS}$, and the wide-gap insulators $\mathrm{LiF}$ and Ar. The strength of the excitonic effects in these materials covers a wide range, with the exciton binding energy $E_{\mathrm{b}}$ varying over three orders of magnitude. Furthermore, CdS was chosen to give an example for systems containing transition metals.

\section{Exciton binding energies}

Table I shows $E_{\mathrm{b}}$ for the materials mentioned above, calculated with various approaches and compared to experiment. The results are also graphically illustrated in Fig. 1.

Compared to experiment, the standard BSE tends to systematically overbind the excitons. There are several reasons for this: mainly the k-point sampling, but also underestimation of dielectric screening for some materials and exclusion of dynamic effects for both band structures and optical properties. We present the evolution of $E_{\mathrm{b}}$ depending on the number of k points in Appendix A, which shows the same convergence behavior for all methods under study. According to Ref. [4],

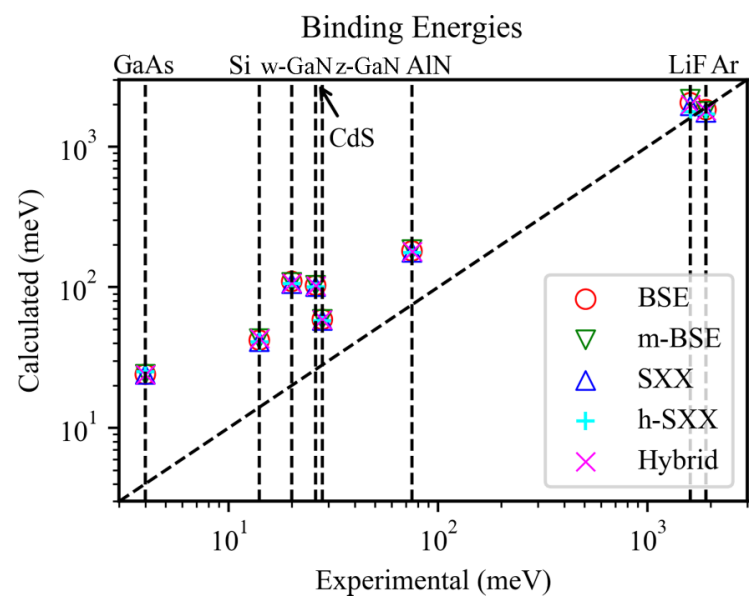

FIG. 1. Exciton binding energies $E_{\mathrm{b}}$ calculated with different variants of BSE and SXX for GaAs, Si, z-GaN, w-GaN, CdS, AlN, $\mathrm{LiF}$, and $\mathrm{Ar}$, compared with experimental values. for the excitons in GaAs one would have to increase the $\mathbf{k}$-point grid density to $10^{8} \mathbf{k}$ points in the Brillouin zone to reach the limits of numerical precision, which is obviously unaffordable.

However, our goal is not to reproduce the experimental $E_{\mathrm{b}}$ to the maximal level of attainable precision, but to demonstrate that the simplified BSE approaches are good approximations for the full BSE. In other words, we here compare theory with theory, taking the full BSE at a given (affordable) level of numerical precision as the benchmark for the other, more approximate, methods.

For all semiconductors considered, we find that the $E_{\mathrm{b}}$ calculated with m-BSE, SXX, and the hybrid functional are remarkably close to the BSE results (to within a couple of meV for GaAs, Si, CdS, and $\mathrm{GaN}$ and to within $10 \mathrm{meV}$ for AlN), which demonstrates the usefulness of these approaches.

The $E_{\mathrm{b}}$ calculated with h-SXX are practically identical to those obtained with SXX, except for LiF and Ar. This indicates that the influence of the LFEs on the excitons is negligible in most covalent semiconductors, and only the long-range electron-hole interaction is relevant. By contrast, in ionic insulators such as $\mathrm{LiF}$ and wide-gap materials such as solid Ar, the Frenkel-type excitons are tightly bound around the ions. Although the long-range excitonic interactions are still dominant, the diagonal short-range interaction and LFEs play a much more important role than for semiconductors. In the following, we focus on LiF; the behavior in Ar is similar.

We found that $E_{\mathrm{b}}$ of $\mathrm{LiF}$ calculated with m-BSE $(2.17 \mathrm{eV})$ is larger than that of the BSE $(2.05 \mathrm{eV})$. This overestimation by $\mathrm{m}$-BSE can be mainly attributed to the neglect of the off-diagonal $\mathbf{G}, \mathbf{G}^{\prime}$ terms of the dielectric function. To confirm this, we considered a diagonal BSE (d-BSE) by setting $\mathbf{G}=$ $\mathbf{G}^{\prime}$ in Eq. (5), which also results in a larger $E_{\mathrm{b}}(2.25 \mathrm{eV})$ compared to the BSE.

On the other hand, the diagonal terms in the dielectric function are even more critical. Compared with the m-BSE, SXX reduces $E_{\mathrm{b}}$ by $0.24 \mathrm{eV}$. A main reason for this is that $\mathrm{SXX}$ does not consider the decay of the dielectric function depending on $\mathbf{q}$, and thus the diagonal short-range electron-hole interactions in $\mathrm{LiF}$ are overscreened. Nevertheless, our results shows that one can still use m-BSE and SXX to calculate $E_{\mathrm{b}}$ in insulators with moderate deviations from the full BSE.

According to the discussion above, the LFEs play a significant role for ionic insulators. In our hybrid functional, the LFEs are included via the ALDA kernel. For LiF (see Table I), $E_{\mathrm{b}}$ calculated with the hybrid functional is $2.00 \mathrm{eV}$, which deviates by only $0.05 \mathrm{eV}$ from $E_{\mathrm{b}}$ by the BSE; by comparison, $E_{\mathrm{b}}$ by m-BSE deviates by $0.12 \mathrm{eV}$ from the BSE. The hybrid functional corrects not only the diagonal shortrange interaction but also the off-diagonal LFE. Therefore, one can expected our hybrid functional to generally perform well for excitons in insulators.

\section{Convergence with the number of $\mathrm{G}$ vectors}

The computational cost of the BSE and its simplified versions depends not only on the complexity of the kernel $K^{\mathrm{x}}$, i.e., whether the full inverse dielectric matrix $\varepsilon_{\mathbf{G G}^{\prime}}^{-1}$ is required, only the diagonal elements of it, or just a simple model such as $\varepsilon(q)$ or $\gamma$, but also on the number of reciprocal lattice vectors 

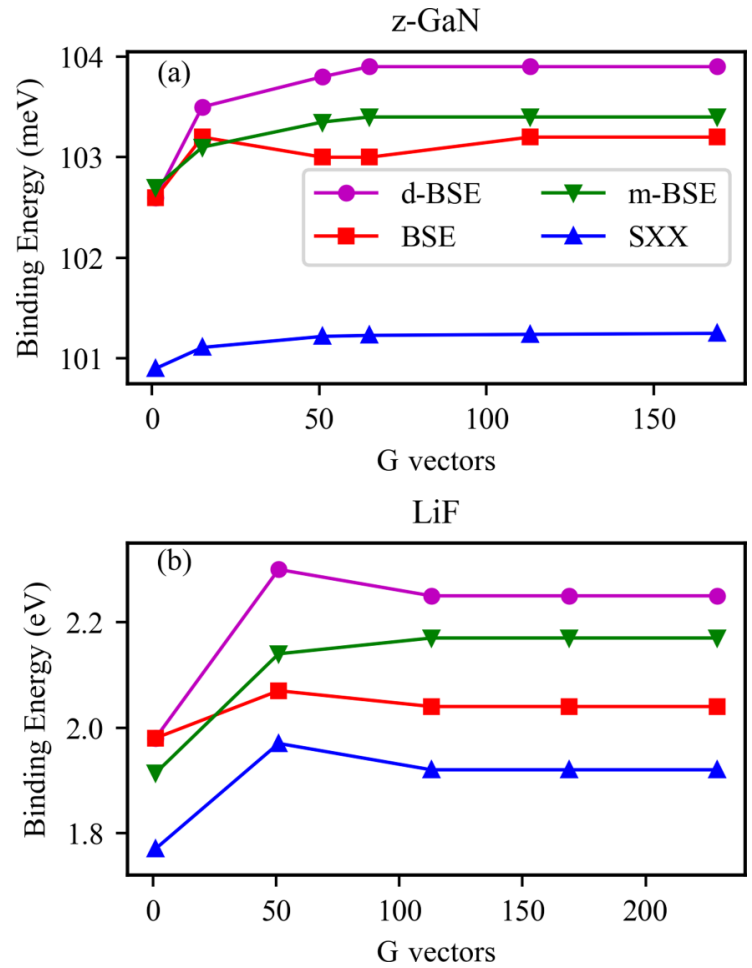

FIG. 2. The G-vector-dependent convergence behavior of $E_{\mathrm{b}}$ in d-BSE, BSE, m-BSE, and SXX, for $\mathrm{z}-\mathrm{GaN}$ and $\mathrm{LiF}$.

$N_{\mathbf{G}}$ which are needed to achieve converged results. Thus, even if a method is formally simpler than the BSE, a large $N_{\mathbf{G}}$ could make it computationally unfavorable.

To test the convergence with respect to $N_{\mathbf{G}}$, we take $\mathrm{z}-\mathrm{GaN}$ and $\mathrm{LiF}$ as representative examples for semiconductors and insulators with direct band gaps and well-separated excitonic peaks in the optical spectrum. We consider $E_{\mathrm{b}}$ to be converged if it changes by less than 0.5 and $5 \mathrm{meV}$ for $\mathrm{z}-\mathrm{GaN}$ and $\mathrm{LiF}$, respectively. The dependence of $E_{\mathrm{b}}$ on $N_{\mathrm{G}}$ is plotted in Fig. 2 for the two materials. In addition to the BSE, m-BSE, and SXX, we also considered d-BSE (only diagonal elements in the BSE kernel). We did not test the $N_{\mathbf{G}}$ dependence for the hybrid kernel, since its screened exchange part is the same as SXX and its ALDA part is treated in the same way as the direct kernel [see Eqs. (3) and (13)].

For z-GaN, all four methods show a quick convergence, and we find that $51 \mathbf{G}$ vectors are enough. For $\mathrm{LiF}$, on the other hand, $169 \mathbf{G}$ vectors are needed. The different convergence of $\mathrm{z}-\mathrm{GaN}$ and $\mathrm{LiF}$ is mainly due to the stronger bound excitonic effects in LiF, which makes the short-range interactions more critical. This can be clearly seen by comparing m-BSE and d-BSE in LiF: The differences seen in Fig. 2(b) result from the differences between the model and RPA dielectric functions (see Appendix $\mathrm{C}$ for more details).

It is also interesting that the finally converged $E_{\mathrm{b}}\left(N_{\mathbf{G}}=\right.$ $169)$ using d-BSE differs from the initial $E_{\mathrm{b}}\left(N_{\mathbf{G}}=0\right)$ by at least $120 \mathrm{meV}$, but the corresponding difference for the BSE is less than $90 \mathrm{meV}$ [see Fig. 2(b)]. A similar but less pronounced tendency is found in z-GaN. This can clearly be attributed to the effects of the off-diagonal terms in the dielectric screening.
TABLE II. SXX, m-BSE, and hybrid exciton binding energies $E_{\mathrm{b}}$, with inverse dielectric constants $\left(\varepsilon^{\mathrm{RPA}}\right)_{0,0}^{-1}$ varied within a $10 \%$ range, for $\mathrm{z}-\mathrm{GaN}$ (in $\mathrm{meV}$ ) and $\mathrm{LiF}$ (in $\mathrm{eV}$ ).

\begin{tabular}{|c|c|c|c|c|c|c|}
\hline Approach & $+10 \%$ & $\begin{array}{c}\mathrm{z}-\mathrm{GaN} \\
\left(\varepsilon^{\mathrm{RPA}}\right)_{0,0}^{-1}\end{array}$ & $-10 \%$ & $+10 \%$ & $\begin{array}{c}\mathrm{LiF} \\
\left(\varepsilon^{\mathrm{RPA}}\right)_{0,0}^{-1}\end{array}$ & $-10 \%$ \\
\hline SXX & 92 & 101 & 112 & 1.63 & 1.93 & 2.34 \\
\hline m-BSE & 93 & 103 & 114 & 1.87 & 2.17 & 2.57 \\
\hline hybrid & 92 & 102 & 113 & 1.68 & 2.00 & 2.42 \\
\hline
\end{tabular}

Regardless of the details above, we find for both smalland large-gap materials that the number of $\mathbf{G}$ vectors needed for convergence is similar for the BSE, m-BSE, SXX, and the hybrid kernel.

\section{Variation of the screening parameter}

The screened Coulomb interaction $W$ in Eq. (5) is determined by the RPA dielectric function, which requires the Kohn-Sham band structure as input. Moreover, $\varepsilon_{0,0}^{\mathrm{RPA}}$ is usually used to obtain the macroscopic dielectric constant as $\varepsilon_{\mathrm{M}} \approx$ $1 /\left(\varepsilon^{\mathrm{RPA}}\right)_{0.0}^{-1}$. As mentioned before, one can also manually adjust $\varepsilon(0)$ or $\gamma$ in m-BSE, SXX, and in the hybrid functional, in the spirit of an empirical calculation.

The accuracy of $1 /\left(\varepsilon^{\mathrm{RPA}}\right)_{0,0}^{-1}$ relies on the Kohn-Sham band structure, and therefore on the DFT approximation used; on top of this, convergence with the number of $\mathbf{k}$ points and unoccupied bands may sometimes be difficult to achieve for reasons of computational cost. This raises the question of how sensitive the calculated excitonic effects are to variations of the dielectric constant. From prior experience $[27,87]$ it is known that $1 /\left(\varepsilon^{\mathrm{RPA}}\right)_{0,0}^{-1}$ can differ from $\varepsilon_{\mathrm{M}}$ by about $10 \%$. In the following, we will perform tests to see how $E_{\mathrm{b}}$ is influenced by fluctuations of the dielectric screening.

In Table II we list the calculated $E_{\mathrm{b}}$ via m-BSE, SXX, and the hybrid functional with $1 / \varepsilon(0)=\gamma=\left(\varepsilon^{\mathrm{RPA}}\right)_{0,0}^{-1}$ and $\left(\varepsilon^{\mathrm{RPA}}\right)_{0,0}^{-1} \pm 10 \%$ for $\mathrm{z}-\mathrm{GaN}$ and $\mathrm{LiF}$. In the case of $\mathrm{z}-\mathrm{GaN}$, $+10 \%$ and $-10 \%\left(\varepsilon^{\mathrm{RPA}}\right)_{0,0}^{-1}$ lead to $90 \%$ and $112 \% E_{\mathrm{b}}$, respectively. This suggests that for small-gap materials the exciton binding energy changes linearly under variation of $\left(\varepsilon^{\mathrm{RPA}}\right)_{0,0}^{-1}$ in the $10 \%$ range for all three methods considered.

This linearity no longer quite holds for LiF, and there appears to be a greater sensitivity of the excitonic effects to variations of the screening parameters. With a $10 \%$ increase of $\left(\varepsilon^{\mathrm{RPA}}\right)_{0,0}^{-1}, E_{\mathrm{b}}$ decreases by $0.3 \mathrm{eV}$ (i.e., by about $15 \%$ ) for all three methods. Under a $10 \%$ decrease of $\left(\varepsilon^{\mathrm{RPA}}\right)_{0,0}^{-1}$, $E_{\mathrm{b}}$ reacts more strongly and increases by $0.4 \mathrm{eV}$. We found the hybrid functional a little more sensitive (see Table II). Due to the negative sign of $K^{\mathrm{SXX}}$ in Eq. (12), the screened part of the ALDA $\left(-\gamma K_{\mathrm{xc}}^{\mathrm{ALDA}}\right)$ actually amplifies the response of $K^{\mathrm{SXX}}$ to the variation of $\gamma$. However, since $K_{\mathrm{xc}}^{\mathrm{ALDA}}$ contributes much less to the dominant long-range interaction, the hybrid functional shows a sensitivity similar to SXX for both $\mathrm{z}-\mathrm{GaN}$ and $\mathrm{LiF}$.

We note that in Eq. (8), the parameter $\varepsilon(0)$ is defined as the dielectric constant. However, the dielectric constant from the RPA $\left(\varepsilon_{0,0}^{\mathrm{RPA}}\right)$ differs a little from $1 /\left(\varepsilon^{\mathrm{RPA}}\right)_{0,0}^{-1}$. It should be obtained by strictly computing the inverse of the matrix 
$\left(\varepsilon^{\mathrm{RPA}}\right)_{\mathbf{G}, \mathbf{G}^{\prime}}^{-1}$ in Eq. (6) and then taking the head of the new matrix as

$$
\varepsilon_{0,0}^{\mathrm{RPA}}=\left[\left(\varepsilon^{\mathrm{RPA}}\right)_{\mathbf{G}, \mathbf{G}^{\prime}}^{-1}\right]_{\mathbf{q}=\mathbf{G}=\mathbf{G}^{\prime}=0}^{-1},
$$

where the LFEs are included in the dielectric constant. Using Eq. (14), we obtained $\varepsilon_{0,0}^{\mathrm{RPA}}=5.8$ for $\mathrm{z}-\mathrm{GaN}$, which is $10 \%$ larger than $1 /\left(\varepsilon^{\mathrm{RPA}}\right)_{0,0}^{-1}=5.3$. According to Table II, $E_{\mathrm{b}}$ will be $114 \mathrm{meV}$ if $\varepsilon(0)=\varepsilon_{0,0}^{\mathrm{RPA}}=5.8$ for m-BSE. Although one should use $\varepsilon(0)=\varepsilon_{0,0}^{\mathrm{RPA}}$ for calculating the dielectric function in Eq. (8), the results by m-BSE with $1 /\left(\varepsilon^{\mathrm{RPA}}\right)_{0,0}^{-1}$ applied are in close agreement with those by the BSE. The reason is that by substituting $1 /\left(\varepsilon^{\mathrm{RPA}}\right)_{0,0}^{-1}$ for $\varepsilon(0)$ in Eq. (8), one will get $\varepsilon^{-1}(q=0)=\left(\varepsilon^{\mathrm{RPA}}\right)_{0,0}^{-1}$. Our m-BSE calculations therefore reproduce the BSE results. The physical interpretation is to obtain the $\varepsilon^{-1}(q)$ for dielectric screening for $W_{\mathbf{G}, \mathbf{G}^{\prime}}$; the LFEs (off-diagonal terms) actually have to be considered again as the opposite process of Eq. (14). Therefore, $\varepsilon(0)=1 /\left(\varepsilon^{\mathrm{RPA}}\right)_{0,0}^{-1}$ makes more sense and offers closer results to the BSE when estimating the dielectric screening for excitons.

\section{Optical spectra}

Besides the exciton binding energy, it is important to evaluate the overall shape of the optical spectrum. The spectra of $\mathrm{z}$-GaN, obtained with a $16^{3} \Gamma$-centered uniform k-point mesh using the m-BSE, SXX, hybrid functional, and BSE, are shown in Fig. 3(a). The BSE and m-BSE result in very similar spectra except for some minor differences in the higher-energy range after $5 \mathrm{eV}$. Compared to the BSE, SXX and the hybrid functional reproduce all the main peaks, but with an overall smaller oscillator strength.

However, the $16^{3} \mathbf{k}$-point mesh is not dense enough. The optical spectra calculated with the double k-grid method are plotted in Fig. 3(b). The calculated curves are much more smooth than those in Fig. 3(a) and are closer to experiment. The SXX spectrum matches the experiment best among all the methods, but this may be fortuitous and might not be the case in other materials. In fact, the main source of error in the BSE spectra is that we completely excluded dynamical effects by using a scissors operator for the QP energies and setting $\omega=0$ in the BSE dielectric screening. The dynamical effects due to the self-energy influence both the (single) QP renormalization and the excitonic interaction [62]. If the QP energies are obtained by the $G W$ method, we are able to reproduce the experimental spectrum much better (see Appendix B).

For LiF, we only use a $16^{3} \mathbf{k}$-point mesh, which is already sufficient for obtaining a reasonable shape of the spectrum. As shown in Fig. 4, the BSE predicts a lower excitation energy than the experiment for the first exciton, in agreement with prior studies $[4,5,89]$. The first excitonic peak using SXX differs from the BSE in position and height. Due to the stronger screening in SXX, the calculated peak slightly moves towards the experimental peak, along with a smaller oscillator strength. The m-BSE shifts the exciton to an even lower energy, but reproduces the height of the BSE peak. As expected, the hybrid functional agrees best with the BSE.
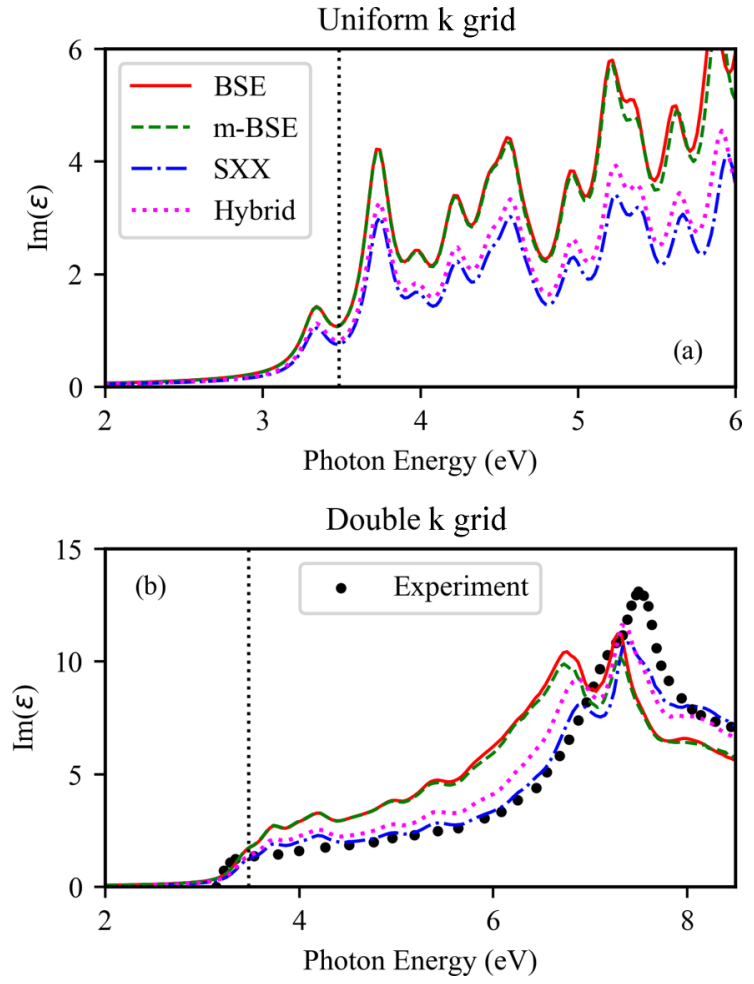

FIG. 3. Optical spectra for z-GaN, calculated by the BSE, mBSE, SXX, and the hybrid functional with (a) uniform $\left(16^{3}\right) \mathbf{k}$ grid and (b) double $\mathbf{k}$ grid $\left(12^{3}+20000\right.$ random). The dashed line shows the experimental band gap. For all spectra, a $0.1-\mathrm{eV}$ broadening was applied. The experimental data are taken from Ref. [77].

\section{B. Application in cubic $\mathrm{CsPbBr}_{3}$}

The reduced computational cost makes m-BSE and SXX attractive for studying excitons in more complex systems. Here we take one of the simplest perovskites, cubic $\mathrm{CsPbBr}_{3}$, as an example of a material where the spin-orbit coupling (SOC) must be included. To calculate the Kohn-Sham band structure, we use a $6 \times 6 \times 6 \Gamma$-centered uniform $\mathbf{k}$ point mesh, along with the Perdew-Burke-Ernzerhof (PBE)

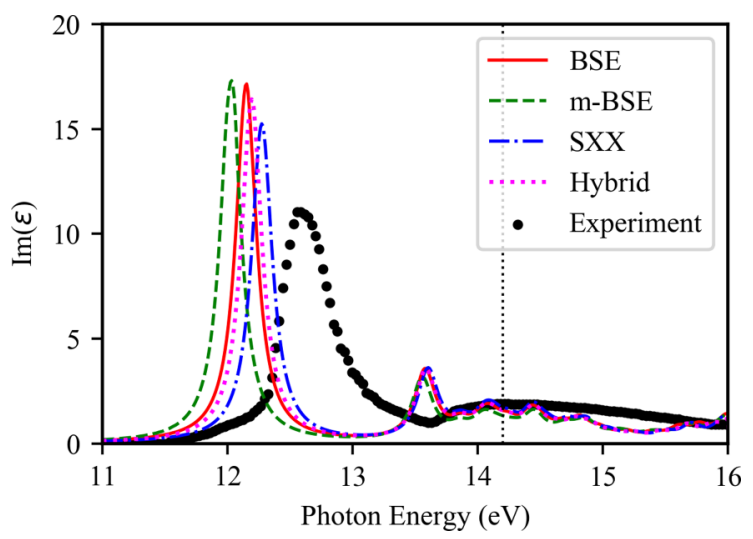

FIG. 4. Optical spectra for LiF, calculated by the BSE, m-BSE, $\mathrm{SXX}$, and the hybrid kernel with a $16^{3} \mathbf{k}$ grid. The dashed line shows the experimental band gap. For all spectra, a $0.1-\mathrm{eV}$ broadening was applied. The experimental data are taken from Ref. [88]. 


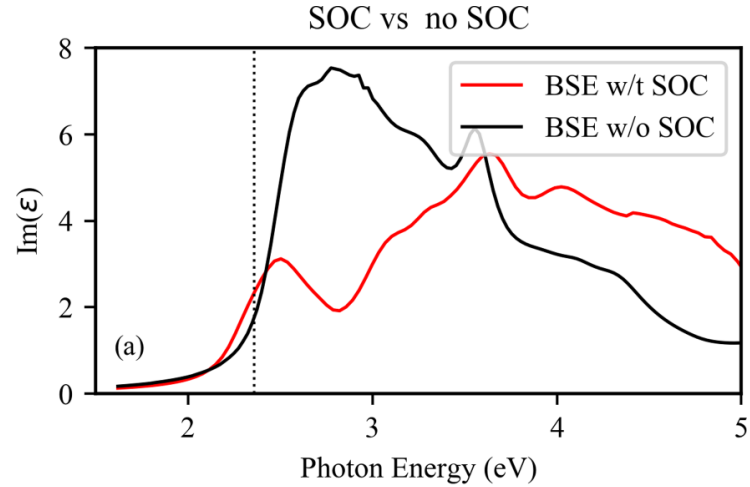

All with SOC

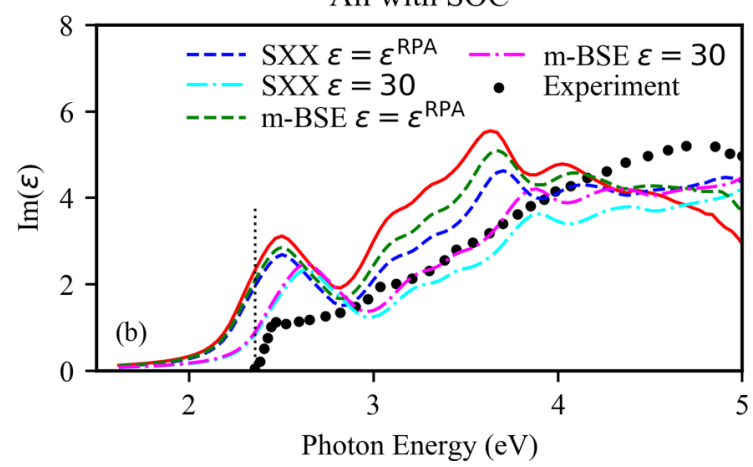

FIG. 5. (a) BSE optical spectra of $\mathrm{CsPbBr}_{3}$ with and without SOC. (b) Comparison of the BSE (solid line), SXX and m-BSE with $\varepsilon^{\mathrm{RPA}}$ (dashed lines), and SXX and m-BSE with $\varepsilon=30$ (dash-dotted lines). For all the spectra, SOC was included and a $0.1-\mathrm{eV}$ broadening was applied. The experimental data are taken from Ref. [93]. The vertical dashed lines show the experimental electronic band gap.

functional [90]. We obtain a Kohn-Sham band gap of $0.54 \mathrm{eV}$, in agreement with previous calculations [91]. We then apply a scissors shift of $1.82 \mathrm{eV}$ to generate a QP band gap which reproduces the experimental gap [92]. To keep the degeneracy, we use eight valence bands and eight conduction bands to build the BSE kernels. The Bethe-Salpeter-type equation is then solved by the inversion solver in YAMBO with 7500 random interpolated $\mathbf{k}$ points. We do not use the hybrid functional for $\mathrm{CsPbBr}_{3}$, because an implementation of SOC in the adiabatic PBE kernel is not available in YAMBO.

It is well known that the strong $\mathrm{SOC}$ from $\mathrm{Pb}$ significantly affects the electronic and optical properties of lead halide perovskites. In Fig. 5(a) we plot the optical spectra of $\mathrm{CsPbr}_{3}$ calculated with and without SOC. There is a huge difference between two spectra, especially in the range of visible light (less than $3.1 \mathrm{eV}$ ). The SOC significantly splits the valence and conduction bands near the Fermi level [94], which reduces the oscillator strength at lower energies. Our results confirm the importance of SOC for optical properties of $\mathrm{CsPBr}_{3}$ on the level of many-body theory. In the following, the SOC is included throughout.

Here we use the experimental data from Ref. [93], which is widely accepted in the literature [95,96], but there are other experiments which produce a much sharper peak at the same position [97-99]. However, the computed perovskite structure is quite sensitive, and the cubic phase we use in this work is actually a high-temperature phase. Thermal vibrations are likely to induce additional broadening effects on the spectrum, which is not included in our calculations.

The calculated excitonic binding energies are 32, 28, and $27 \mathrm{meV}$ by the BSE, m-BSE, and SXX, respectively. These values lie within the range $(2-62 \mathrm{meV})$ quoted in the experimental and theoretical literature [95,96,100,101]. It should be pointed out that these calculated values are obtained with $1 /\left(\varepsilon^{\mathrm{RPA}}\right)_{0,0}^{-1}=5.96$. On the other hand, the experimental dielectric constant of $\mathrm{CsPBr}_{3}$ is reported to be in a wide range from 5 up to 40, though orthorhombic phase samples are used in some works $[97,98,102]$. To account for this, we also calculated $E_{\mathrm{b}}$ with $\varepsilon(0)=1 / \gamma=9,16$, and 30 via m-BSE and SXX. With $\varepsilon(0)=1 / \gamma=9$, we find $E_{\mathrm{b}}=3 \mathrm{meV}$ for both $\mathrm{m}$-BSE and SXX, which agrees with the experimental measurements of Ref. [100]. However, there is no excitation located below the electronic band gap with the other two higher screening parameters of 16 and 30. This implies that excitonic effects in $\mathrm{CsPbBr}_{3}$ are well described using static electronic dielectric constants with both m-BSE and SXX, though the lattice screening is also critical to the dielectric response when measured by different experiments. Our results show that the excitons have a stronger dependence on electronic screening [103].

In Fig. 5(b) we compare the BSE, m-BSE, and SXX optical spectra with experiment. As shown in the figure, the m-BSE and SXX spectra, which were calculated from first principles using $1 /\left(\varepsilon^{\mathrm{RPA}}\right)_{0,0}^{-1}=5.96$, are similar to the BSE in the range of visible light. All three methods successfully reproduce the excitonic peak just above the band gap, though the calculated peaks are much more pronounced than the experimental one. We mention that the single-particle optical spectrum (see Ref. [94]) smoothly increases without any obvious excitonic peak.

There are two main differences between the SXX and BSE spectra. The absorption strength by SXX is not as strong as with the BSE, which we already observed in $\mathrm{z}-\mathrm{GaN}$. The other difference is the spectrum for excitation energy above $3.5 \mathrm{eV}$. The imaginary part of the BSE dielectric function decreases beyond $4 \mathrm{eV}$, which is not observed in the experiment. On the other hand, the SXX spectrum comes to a sharp drop around $4.9 \mathrm{eV}$, which is in agreement with the experiment.

One potential reason for the behavior of the BSE is the effect of free electrons on the dielectric function, which can be simply expressed as $\varepsilon\left(q, n_{\mathrm{c}}\right)=\varepsilon_{\infty}\left(1+\frac{q_{\mathrm{TF}}^{2}}{q^{2}}\right)$, where the Thomas-Fermi wave vector is $q_{\mathrm{TF}} \propto \sqrt{n_{\mathrm{c}}}$. Here $n_{\mathrm{c}}$ is the concentration of free electrons in the system. However, $n_{\mathrm{c}}$ may be seriously underestimated by our model, since we did not consider free electrons arising from defects or donors in the experimental sample. The high free-electron concentration is known to play a critical role in the optical properties in $\mathrm{CH}_{3} \mathrm{NH}_{3} \mathrm{PbBr}_{3}$ perovskites [104]. Since a higher $n_{\mathrm{c}}$ leads to a larger $\varepsilon\left(q, n_{\mathrm{c}}\right)$, we get $\left(\varepsilon^{\mathrm{RPA}}\right)^{-1}(q) \geqslant \varepsilon^{-1}\left(q, n_{\mathrm{c}}\right)$, which is similar to the relationship between the dielectric screening of the BSE and SXX, where $\left(\varepsilon^{\mathrm{RPA}}\right)_{\mathbf{G}, \mathbf{G}^{\prime}}^{-1}(q) \geqslant \gamma=\varepsilon_{0,0}^{-1, \mathrm{RPA}}$. Hence, we find the SXX optical spectrum to be closer to experiment.

Since the experimental dielectric constant varies over a rather wide range from 5 up to 40 [97,98,102], we also plotted the optical spectra calculated with $\varepsilon(0)=$ $1 / \gamma=30$ in Fig. 5 (b). Compared with $1 /\left(\varepsilon^{\mathrm{RPA}}\right)_{0,0}^{-1}=5.96$, 
dielectric screening becomes much stronger with $\varepsilon(0)=$ $1 / \gamma=30$, bringing two significant changes for both $\mathrm{m}-\mathrm{BSE}$ and SXX. Not only is the first peak significantly blueshifted, but also the optical absorption strength becomes lower. The continuum spectrum beyond $3.0 \mathrm{eV}$ provides a better match with the experiment when $\varepsilon(0)=1 / \gamma=30$. Further improvement could be achieved by carefully choosing $\varepsilon(0)$ and tuning $\alpha$ in Eq. (8) [17], as well as including the free-electron effects. In addition, the optical spectrum is expected to be improved by considering the dynamic effects with a QP band structure as we mentioned for $\mathrm{z}-\mathrm{GaN}$.

Finally, we have assessed the computational cost of our approaches for the case of the perovskite. In Appendix D we compare the CPU times used by the BSE and SXX and find that SXX and m-BSE lead to quite a significant speedup of up to two orders of magnitude, depending on the details of the calculation.

\section{SUMMARY}

The central goal of this paper was to find simplifications of the Bethe-Salpeter equation for calculating optical spectra of insulators and semiconductors, with a particular emphasis on capturing spectral properties, including excitons, close to the optical gap. The main focus was on reducing the computational effort that goes into constructing the screened Coulomb interaction, which is the centerpiece of the BSE approach. In the BSE, the frequency- and wave-vector-dependent dielectric function (formally a matrix in the space of reciprocal lattice vectors) must be computed from first principles using the RPA. We found that the dielectric function can be replaced by simple models, or even a single screening parameter, without significant loss of accuracy. The gain in computational speed achieved in this way can be considerable.

We combined the simplified BSE kernel (referred to as screened exact exchange) with a local xc kernel, which constitutes a simple hybrid functional in the context of generalized TDDFT. We then performed a detailed numerical assessment of our simplified BSE schemes and the hybrid approach for several elemental and binary materials, including the widegap insulators $\mathrm{LiF}$ and Ar. We also studied a perovskite material as an example of a more complex system. We found that all methods produce exciton binding energies and optical spectra in close agreement with the full BSE.

In DFT, hybrid functionals are becoming increasingly popular in materials science since they provide an efficient way to approximate the quasiparticle band structure, which leads to much improved band gaps. In TDDFT for solids, hybrid functionals are now also beginning to be used [50-56], and there are many indications that hybrids may be the most promising approach to describe excitons from first principles.

In this paper we showed that hybrid functionals directly follow from a suitably simplified BSE. Our construction is a very simple one, where the admixture of screened exact exchange and semilocal exchange and correlation is governed by a single parameter $\gamma$. This parameter can be calculated using the RPA, which makes the hybrid approach completely nonempirical; but it can also be taken from experiment, or fitted to reproduce reference data. Clearly, more sophisticated constructions such as multiparameter or local hybrids are possible, and the recent history of DFT shows that such efforts, combined with systematic assessments using materials databases or test sets, can be extremely fruitful.

\section{ACKNOWLEDGMENTS}

This work was supported by NSF Grant No. DMR1810922. The computation for this work was performed on the high performance computing infrastructure provided by Research Computing Support Services at the University of Missouri-Columbia. J.Y. acknowledges support from the National Natural Science Foundation of China (Grant No. 21688102), the National Key Research \& Development Program of China (Grant No. 2016YFA0200604), and Anhui Initiative in Quantum Information Technologies (Grant No. AHY090400). We thank Matteo Gatti and Sahar Sharifzadeh for valuable comments.

\section{APPENDIX A: DEPENDENCE OF EXCITON BINDING ENERGY ON k-POINT SAMPLING}

We use the QUANTUM ESPRESSO code [68] for the electronic band structures and the YAMBO code [72] for the electronic excitation spectra. In both codes we use uniform k-point sampling. Here we give an example of the dependence of the exciton binding energy on the number of $\mathbf{k}$ points, for the case of $\mathrm{z}-\mathrm{GaN}$. The calculations are done with the full BSE, $\mathrm{m}-\mathrm{BSE}$, and SXX.

Figure 6 shows the exciton binding energy $E_{\mathrm{b}}$ plotted as a function of the number of $\mathbf{k}$ points $N_{k}$ for grid sizes ranging from $16 \times 16 \times 16$ up to $30 \times 30 \times 30$. For all the tested methods (the BSE, m-BSE, and $\mathrm{SXX}$ ), $E_{\mathrm{b}}$ shows a quite similar behavior depending on the k-point sampling density: $E_{b}$ decreases monotonically from around $150 \mathrm{meV}$ for $16^{3} \mathbf{k}$ points down to $80 \mathrm{meV}$ for $30^{3} \mathbf{k}$ points. The experimental value is $26 \mathrm{meV}$ (see Table I). From the data shown in Fig. 6 it is clear that $E_{\mathrm{b}}$ is not yet fully converged, which would require a considerably larger number of $\mathbf{k}$ points and would bring the calculation closer to the experimental value.

A similar convergence analysis (for the quasiparticle gap) was also recently carried out for two-dimensional materials by Rasmussen et al. [105]. We also note that Rohlfing and Louie [4] point out that a converged $E_{\mathrm{b}}$ for GaAs would need around

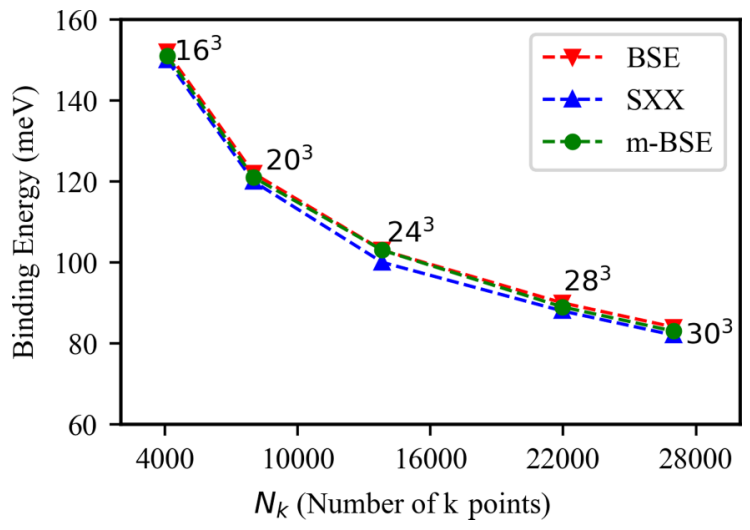

FIG. 6. Exciton binding energy of z-GaN, as a function of the number of $\mathbf{k}$ points, for the BSE, $\mathrm{m}-\mathrm{BSE}$, and SXX. 
$10^{8} \mathbf{k}$ points; however, much fewer $\mathbf{k}$ points are sufficient if they are distributed in such a way that there is a high enough sampling density close to the zone center.

\section{APPENDIX B: QUASIPARTICLE CORRECTIONS TO THE OPTICAL SPECTRUM OF z-GaN}

Figure 3 shows the optical spectra of z-GaN. The BSE calculation deviates from the experimental optical spectrum and one may suspect that this is because we use LDA+scissors electronic band structures. To test the influence of the electronic band structure, we perform a single-shot $G_{0} W_{0}$ calculation to obtain quasiparticle corrections to the Kohn-Sham band structure. The dynamic effects on the quasiparticles are estimated by the generalized plasmon-pole model [65]. The calculations in this section are done with a $12^{3}+20000 \mathbf{k}$ point double grid, which is the same as that for the optical spectra of z-GaN shown in Fig. 3(b).

In Fig. 7(a) we show the energy levels at the $\Gamma$ point for $\mathrm{z}-\mathrm{GaN}$, comparing LDA, LDA+scissors, and $G_{0} W_{0}$. As can be seen, the $G_{0} W_{0}$ calculation produces an electronic band gap of $3.47 \mathrm{eV}$, which is very close to the experimental value of $3.48 \mathrm{eV}$. By construction, the LDA+scissors gap is the same as the experimental one. However, the higher conduction band levels and lower valence band levels are further shifted within $G_{0} W_{0}$ compared to LDA+scissors.

As shown in Fig. 7(b), the $G_{0} W_{0}$ band structure leads to a BSE optical spectrum that is much closer to experiment. The $G_{0} W_{0}+\mathrm{m}$-BSE produces a spectrum very similar to $G_{0} W_{0}+\mathrm{BSE}$, in agreement with Fig. 3(b). It also shows that
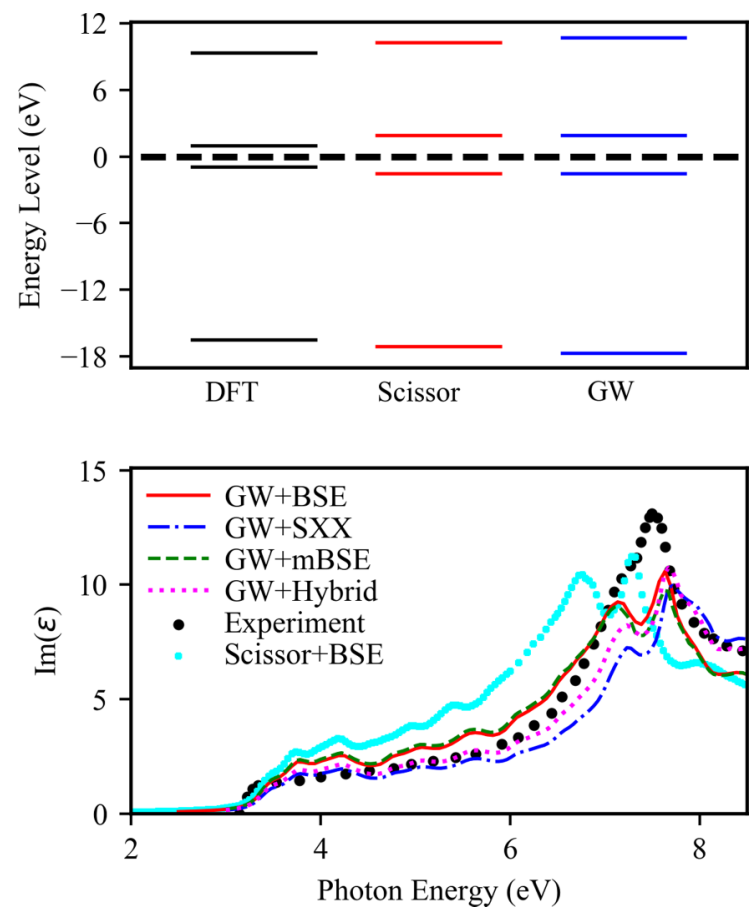

FIG. 7. (a) Energy levels at the $\Gamma$ point for $z-G a N$, calculated using the LDA, LDA+scissors, and $G_{0} W_{0}$. (b) Optical spectra for $\mathrm{z}-\mathrm{GaN}$, calculated using the BSE with the LDA+scissors and $G_{0} W_{0}$ band structures. The optical spectra using m-BSE, SXX, and the hybrid functional on top of $G_{0} W_{0}$ are also shown.

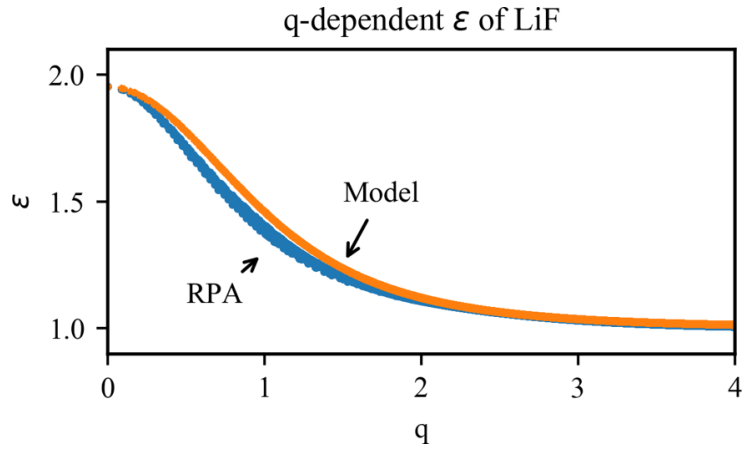

FIG. 8. The $q$-dependent dielectric function of LiF calculated from first principles using the RPA as the diagonal of $\varepsilon_{\mathbf{G}, \mathbf{G}^{\prime}}(q)$ and calculated using the model by Cappellini et al. [13].

SXX with $G_{0} W_{0}$ underestimates the spectral strength, which is hardly surprising since SXX, by construction, tends to overestimate the screening. As expected, the hybrid functional kernel with $G_{0} W_{0}$ improves the results of SXX, resulting in a spectrum of similar quality to the $G_{0} W_{0}+$ m-BSE spectrum.

We also calculated the exciton binding energy $E_{\mathrm{b}}$ using the $G_{0} W_{0}$ band structure and found a value of $110 \mathrm{meV}$, which is only $5 \mathrm{meV}$ larger than $E_{\mathrm{b}}$ by using the LDA+scissors band structure (105 meV, using the same double k-point grid). This suggests that using a quasiparticle band structure makes less of a difference when looking at excitonic effects close to the band edge.

\section{APPENDIX C: DIELECTRIC FUNCTION OF LiF}

Table I compares exciton binding energies $E_{\mathrm{b}}$ calculated with different approaches (the BSE, m-BSE, SXX, h-SXX, and hybrid). It is found that all methods produce very similar results, except for the wide-gap insulators $\mathrm{LiF}$ and (to a somewhat lesser extent) Ar.

In the case of $\mathrm{LiF}$, local-field effects play a significant role in the exciton binding, especially the diagonal element of $\varepsilon_{\mathbf{G}, \mathbf{G}^{\prime}}^{-1}(\mathbf{q})$. We compared a version of the BSE where only the diagonal elements of the full $\varepsilon_{\mathbf{G}, \mathbf{G}^{\prime}}^{-1}(\mathbf{q})$ are included (d-BSE) with $\mathrm{m}-\mathrm{BSE}$, which uses the (diagonal) model dielectric function by Cappellini et al. [13] [see Eq. (8)].

The two momentum-dependent dielectric functions are compared for LiF in Fig. 8. It can be seen that the model $\varepsilon(q)$ lies slightly above the RPA dielectric function, which explains the fact that $E_{\mathrm{b}}$ calculated with m-BSE $(2.17 \mathrm{eV})$ is found to be lower than that calculated with d-BSE $(2.25 \mathrm{eV})$, due to the slightly stronger screening.

\section{APPENDIX D: COMPUTATIONAL SPEEDUP OF THE SIMPLIFIED BSE}

In the main text we pointed out that the principal motivation for simplifying the BSE is to speed up the calculation. To estimate the speedup, we have performed a comparison of the CPU times between SXX and BSE for calculating the exciton 


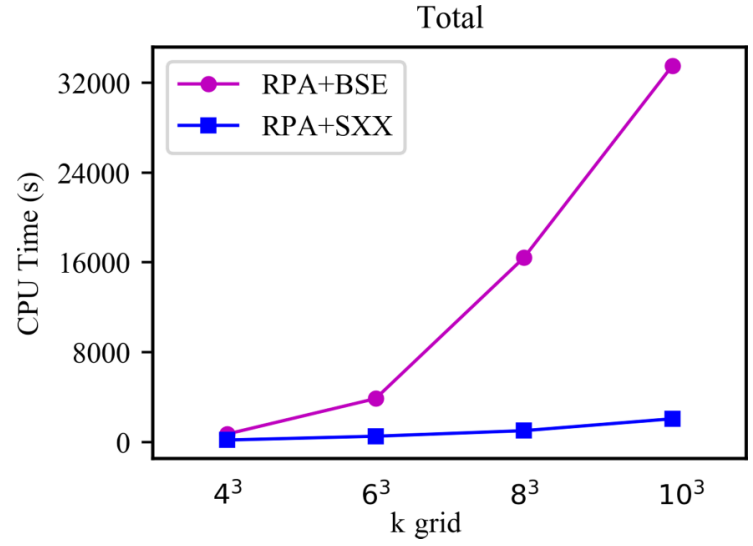

FIG. 9. Total CPU time for calculating the exciton binding energy of $\mathrm{CsPbBr}_{3}$ using $\mathrm{SXX}$ and BSE.

binding energy of $\mathrm{CsPbBr}_{3}$. The computational details are the same as those given in Sec. III B. Here we vary the k-grid size from $4^{3}$ to $10^{3}$.

For larger systems, the main bottleneck of doing BSEtype calculations lies in the memory requirements. Therefore, we have carried out our calculations (using version 4.3.2 of YAMBO) with a configuration that is optimized towards using the least amount of memory, instead of the most CPU efficient one. The same configuration is used for the SXX calculations. The results are shown in Figs. 9 and 10.

Figure 9 gives the CPU time for the total calculation, which includes building the kernel $K^{\mathrm{x}}(\mathbf{q}$,$) [Eq. (4)] and solving the$ Tamm-Dancoff equation (2) to obtain $E_{\mathrm{b}}$. Clearly, SXX becomes vastly more efficient with increasing grid size (almost

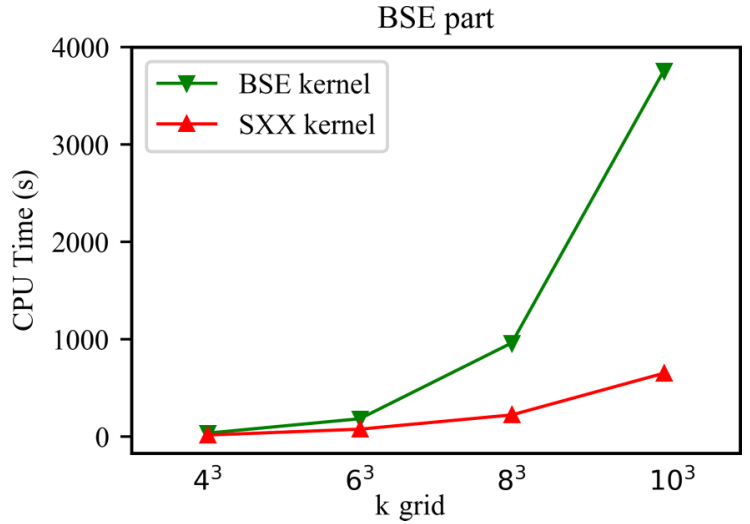

FIG. 10. Same as Fig. 9 but only the CPU time spent after calculating the screened Coulomb interaction.

two orders of magnitude for $10^{3}$ ). The acceleration in SXX mainly comes from not having to calculate the full inverse dielectric function $\left(\varepsilon^{\mathrm{RPA}}\right)_{\mathbf{G}, \mathbf{G}^{\prime}}^{-1}(\mathbf{q})$, but instead only doing a single-point RPA calculation to obtain the RPA dielectric constant $\varepsilon(0)=1 /\left(\varepsilon^{\mathrm{RPA}}\right)_{0,0}^{-1}(0)$.

This is confirmed in Fig. 10, which illustrates the remaining computational cost after building the kernel $K^{\mathrm{x}}(\mathbf{q})$. The SXX acceleration now comes from having $\delta_{\mathbf{G}, \mathbf{G}^{\prime}}$ in the screened Coulomb interaction. Clearly, this is only a fraction of the total CPU time shown in Fig. 9.

Therefore, we conclude that the RPA calculation of the full dielectric function is the most costly part for a doing a BSE calculation in a complex system like $\mathrm{CsPBBr}_{3}$, and our simplifications (m-BSE, SXX, and hybrid) take full advantage of this.
[1] W. Hanke and L. J. Sham, Many-particle effects in the optical spectrum of a semiconductor, Phys. Rev. B 21, 4656 (1980).

[2] M. Rohlfing and S. G. Louie, Electron-hole excitations and optical spectra from first principles, Phys. Rev. B 62, 4927 (2000).

[3] G. Onida, L. Reining, and A. Rubio, Electronic excitations: density-functional versus many-body Green's-function approaches, Rev. Mod. Phys. 74, 601 (2002).

[4] M. Rohlfing and S. G. Louie, Electron-Hole Excitations in Semiconductors and Insulators, Phys. Rev. Lett. 81, 2312 (1998).

[5] L. X. Benedict, E. L. Shirley, and R. B. Bohn, Optical Absorption of Insulators and the Electron-Hole Interaction: An Ab Initio Calculation, Phys. Rev. Lett. 80, 4514 (1998).

[6] X. Leng, F. Jin, M. Wei, and Y. Ma, GW method and Bethe-Salpeter equation for calculating electronic excitations, WIREs Comput. Mol. Sci. 6, 532 (2016).

[7] S. L. Adler, Quantum theory of the dielectric constant in real solids, Phys. Rev. 126, 413 (1962).

[8] N. Wiser, Dielectric constant with local field effects included, Phys. Rev. 129, 62 (1963).

[9] R. Del Sole and R. Girlanda, Optical properties of semiconductors within the independent-quasiparticle approximation, Phys. Rev. B 48, 11789 (1993).
[10] D. R. Penn, Wave-number-dependent dielectric function of semiconductors, Phys. Rev. 128, 2093 (1962).

[11] Z. H. Levine and S. G. Louie, New model dielectric function and exchange-correlation potential for semiconductors and insulators, Phys. Rev. B 25, 6310 (1982).

[12] M. S. Hybertsen and S. G. Louie, Model dielectric matrices for quasiparticle self-energy calculations, Phys. Rev. B 37, 2733 (1988).

[13] G. Cappellini, R. Del Sole, L. Reining, and F. Bechstedt, Model dielectric function for semiconductors, Phys. Rev. B 47, 9892 (1993).

[14] F. Bechstedt, R. D. Sole, G. Cappellini, and L. Reining, An efficient method for calculating quasiparticle energies in semiconductors, Solid State Commun. 84, 765 (1992).

[15] M. Palummo, R. D. Sole, L. Reining, F. Bechstedt, and G. Cappellini, Screening models and simplified GW approaches: Si and GaN as test cases, Solid State Commun. 95, 393 (1995).

[16] M. L. Trolle, T. G. Pedersen, and V. Véniard, Model dielectric function for 2D semiconductors including substrate screening, Sci. Rep. 7, 39844 (2017).

[17] J. Luo, X. Wang, S. Li, J. Liu, Y. Guo, G. Niu, L. Yao, Y. Fu, L. Gao, Q. Dong et al., Efficient and stable emission of warmwhite light from lead-free halide double perovskites, Nature (London) 563, 541 (2018). 
[18] E. Runge and E. K. U. Gross, Density-Functional Theory for Time-Dependent Systems, Phys. Rev. Lett. 52, 997 (1984).

[19] M. Petersilka, U. J. Gossmann, and E. K. U. Gross, Excitation Energies from Time-Dependent Density-Functional Theory, Phys. Rev. Lett. 76, 1212 (1996).

[20] C. A. Ullrich, Time-Dependent Density-Functional Theory: Concepts and Applications (Oxford University Press, Oxford, 2012).

[21] R. Del Sole, G. Adragna, V. Olevano, and L. Reining, Long-range behavior and frequency dependence of exchangecorrelation kernels in solids, Phys. Rev. B 67, 045207 (2003).

[22] S. Botti, F. Sottile, N. Vast, V. Olevano, L. Reining, H.-C. Weissker, A. Rubio, G. Onida, R. Del Sole, and R. W. Godby, Long-range contribution to the exchange-correlation kernel of time-dependent density functional theory, Phys. Rev. B 69, $155112(2004)$

[23] S. Sharma, J. K. Dewhurst, A. Sanna, and E. K. U. Gross, Bootstrap Approximation for the Exchange-Correlation Kernel of Time-Dependent Density-Functional Theory, Phys. Rev. Lett. 107, 186401 (2011).

[24] S. Sharma, J. K. Dewhurst, and E. K. U. Gross, in First Principles Approaches to Spectroscopic Properties of Complex Materials, edited by C. D. Valentin, S. Botti, and M. Cococcioni, Topics in Current Chemistry Vol. 347 (Springer, Berlin, 2014), p. 235.

[25] Z.-H. Yang and C. A. Ullrich, Direct calculation of exciton binding energies with time-dependent density-functional theory, Phys. Rev. B 87, 195204 (2013).

[26] C. A. Ullrich and Z.-H. Yang, in Density-Functional Methods for Excited States, edited by N. Ferré, M. Filatov, and M. HuixRotllant, Topics in Current Chemistry Vol. 368 (Springer, Berlin, 2015), p. 185.

[27] Y.-M. Byun and C. A. Ullrich, Assessment of long-rangecorrected exchange-correlation kernels for solids: Accurate exciton binding energies via an empirically scaled bootstrap kernel, Phys. Rev. B 95, 205136 (2017).

[28] Y.-M. Byun and C. A. Ullrich, Excitons in solids from timedependent density-functional theory: Assessing the TammDancoff approximation, Computation 5, 9 (2017).

[29] A. Seidl, A. Görling, P. Vogl, J. A. Majewski, and M. Levy, Generalized Kohn-Sham schemes and the band-gap problem, Phys. Rev. B 53, 3764 (1996).

[30] A. Görling and M. Levy, Hybrid schemes combining the Hartree-Fock method and density-functional theory: Underlying formalism and properties of correlation functionals, J. Chem. Phys. 106, 2675 (1997).

[31] R. Baer and L. Kronik, Time-dependent generalized KohnSham theory, Eur. Phys. J. B 91, 170 (2018).

[32] A. D. Becke, Density-functional thermochemistry. III. The role of exact exchange, J. Chem. Phys. 98, 5648 (1993).

[33] A. D. Becke, Density-functional thermochemistry. IV. A new dynamical correlation functional and implications for exactexchange mixing, J. Chem. Phys. 104, 1040 (1996).

[34] J. P. Perdew, M. Ernzerhof, and K. Burke, Rationale for mixing exact exchange with density functional approximations, J. Chem. Phys. 105, 9982 (1996).

[35] P. J. Stephens, F. J. Devlin, C. F. Chabalowski, and M. J. Frisch, $\mathrm{Ab}$ initio calculation of vibrational absorption and circular dichroism spectra using density functional force fields, J. Phys. Chem. 98, 11623 (1994).

[36] C. Adamo and V. Barone, Toward reliable density functional methods without adjustable parameters: The PBE0 model, J. Chem. Phys. 110, 6158 (1999).

[37] J. Heyd, J. E. Peralta, G. E. Scuseria, and R. L. Martin, Energy band gaps and lattice parameters evaluated with the HeydScuseria-Ernzerhof screened hybrid functional, J. Chem. Phys. 112, 174101 (2005).

[38] I. C. Gerber, J. G. Ángyán, M. Marsman, and G. Kresse, Range separated hybrid density functional with long-range HartreeFock exchange applied to solids, J. Chem. Phys. 127, 054101 (2007).

[39] T. M. Henderson, J. Paier, and G. E. Scuseria, Accurate treatment of solids with the HSE screened hybrid, Phys. Status Solidi B 248, 767 (2011).

[40] M. Jain, J. R. Chelikowsky, and S. G. Louie, Reliability of Hybrid Functionals in Predicting Band Gaps, Phys. Rev. Lett. 107, 216806 (2011).

[41] M. A. L. Marques, J. Vidal, M. J. T. Oliveira, L. Reining, and S. Botti, Density-based mixing parameter for hybrid functionals, Phys. Rev. B 83, 035119 (2011).

[42] Y.-I. Matsushita, K. Nakamura, and A. Oshiyama, Comparative study of hybrid functionals applied to structural and electronic properties of semiconductors and insulators, Phys. Rev. B 84, 075205 (2011).

[43] J. E. Moussa, P. A. Schultz, and J. R. Chelikowsky, Analysis of the Heyd-Scuseria-Ernzerhof density functional parameter space, J. Chem. Phys. 136, 204117 (2012).

[44] C. Friedrich, M. Betzinger, M. Schlipf, S. Blügel, and A. Schindlmayr, Hybrid functionals and GW approximation in the FLAPW method, J. Phys.: Condens. Matter 24, 293201 (2012).

[45] J. H. Skone, M. Govoni, and G. Galli, Self-consistent hybrid functional for condensed systems, Phys. Rev. B 89, 195112 (2014).

[46] J. H. Skone, M. Govoni, and G. Galli, Nonempirical rangeseparated hybrid functionals for solids and molecules, Phys. Rev. B 93, 235106 (2016).

[47] M. Gerosa, C. E. Bottani, L. Caramella, G. Onida, C. Di Valentin, and G. Pacchioni, Electronic structure and phase stability of oxide semiconductors: Performance of dielectricdependent hybrid functional DFT, benchmarked against GW band structure calculations and experiments, Phys. Rev. B 91, 155201 (2015).

[48] Y. Hinuma, Y. Kumagai, I. Tanaka, and F. Oba, Band alignment of semiconductors and insulators using dielectricdependent hybrid functionals: Toward high-throughput evaluation, Phys. Rev. B 95, 075302 (2017).

[49] W. Chen, G. Miceli, G.-M. Rignanese, and A. Pasquarello, Nonempirical dielectric-dependent hybrid functional with range separation for semiconductors and insulators, Phys. Rev. Mater. 2, 073803 (2018).

[50] J. Paier, M. Marsman, and G. Kresse, Dielectric properties and excitons for extended systems from hybrid functionals, Phys. Rev. B 78, 121201(R) (2008).

[51] L. Bernasconi, S. Tomić, M. Ferrero, M. Rérat, R. Orlando, R. Dovesi, and N. M. Harrison, First-principles optical response of semiconductors and oxide materials, Phys. Rev. B 83, 195325 (2011). 
[52] S. Tomić, L. Bernasconi, B. G. Searle, and N. M. Harrison, Electronic and optical structure of wurtzite $\mathrm{CuInS}_{2}$, J. Phys. Chem. C 118, 14478 (2014).

[53] R. Webster, L. Bernasconi, and N. M. Harrison, Optical properties of alkali halide crystals from all-electron hybrid TD-DFT calculations, J. Chem. Phys. 142, 214705 (2015).

[54] S. Refaely-Abramson, M. Jain, S. Sharifzadeh, J. B. Neaton, and L. Kronik, Solid-state optical absorption from optimally tuned time-dependent range-separated hybrid density functional theory, Phys. Rev. B 92, 081204(R) (2015).

[55] L. Kronik and J. B. Neaton, Excited-state properties of molecular solids from first principles, Annu. Rev. Phys. Chem. 67, 587 (2016)

[56] D. Wing, J. B. Haber, R. Noff, B. Barker, D. A. Egger, A. Ramasubramaniam, S. G. Louie, J. B. Neaton, and L. Kronik, Comparing time-dependent density functional theory with many-body perturbation theory for semiconductors: Screened range-separated hybrids and the GW plus Bethe-Salpeter approach, Phys. Rev. Mater. 3, 064603 (2019).

[57] A. Ramasubramaniam, D. Wing, and L. Kronik, Transferable screened range-separated hybrids for layered materials: The cases of $\mathrm{MoS}_{2}$ and h-BN, Phys. Rev. Mater. 3, 084007 (2019).

[58] Z.-H. Yang, F. Sottile, and C. A. Ullrich, A simple screened exact-exchange approach for excitonic properties in solids, Phys. Rev. B 92, 035202 (2015).

[59] R. M. Martin, L. Reining, and D. M. Ceperley, Interacting Electrons: Theory and Computational Approaches (Cambridge University Press, Cambridge, 2016).

[60] T. Sander, E. Maggio, and G. Kresse, Beyond the TammDancoff approximation for extended systems using exact diagonalization, Phys. Rev. B 92, 045209 (2015).

[61] L. X. Benedict and E. L. Shirley, Ab initio calculation of $\epsilon_{2}(\omega)$ including the electron-hole interaction: Application to $\mathrm{GaN}$ and $\mathrm{CaF}_{2}$, Phys. Rev. B 59, 5441 (1999).

[62] A. Marini and R. Del Sole, Dynamical Excitonic Effects in Metals and Semiconductors, Phys. Rev. Lett. 91, 176402 (2003).

[63] I. Reshetnyak, M. Gatti, F. Sottile, and L. Reining, Excitons on a microscopic level: The mixed dynamic structure factor, Phys. Rev. Res. 1, 032010 (2019).

[64] M. S. Hybertsen and S. G. Louie, Electron correlation in semiconductors and insulators: Band gaps and quasiparticle energies, Phys. Rev. B 34, 5390 (1986).

[65] R. W. Godby and R. J. Needs, Metal-Insulator Transition in Kohn-Sham Theory and Quasiparticle Theory, Phys. Rev. Lett. 62, 1169 (1989).

[66] P. Larson, M. Dvorak, and Z. Wu, Role of the plasmon-pole model in the GW approximation, Phys. Rev. B 88, 125205 (2013).

[67] A. Schleife, M. D. Neumann, N. Esser, Z. Galazka, A. Gottwald, J. Nixdorf, R. Goldhahn, and M. Feneberg, Optical properties of $\mathrm{In}_{2} \mathrm{O}_{3}$ from experiment and first-principles theory: Influence of lattice screening, New J. Phys. 20, 053016 (2018).

[68] P. Giannozzi, O. Andreussi, T. Brumme, O. Bunau, M. B. Nardelli, M. Calandra, R. Car, C. Cavazzoni, D. Ceresoli, M. Cococcioni et al., Advanced capabilities for materials modeling with QUANTUM ESPRESSO, J. Phys.: Condens. Matter 29, 465901 (2017).
[69] D. R. Hamann, Optimized norm-conserving Vanderbilt pseudopotentials, Phys. Rev. B 88, 085117 (2013); 95, 239906(E) (2017).

[70] Z. H. Levine and D. C. Allan, Linear Optical Response in Silicon and Germanium Including Self-Energy Effects, Phys. Rev. Lett. 63, 1719 (1989).

[71] X. Gonze and C. Lee, Dynamical matrices, Born effective charges, dielectric permittivity tensors, and interatomic force constants from density-functional perturbation theory, Phys. Rev. B 55, 10355 (1997).

[72] A. Marini, C. Hogan, M. Grüning, and D. Varsano, YAMBO: An ab initio tool for excited state calculations, Comput. Phys. Commun. 180, 1392 (2009).

[73] D. Rocca, Y. Ping, R. Gebauer, and G. Galli, Solution of the Bethe-Salpeter equation without empty electronic states: Application to the absorption spectra of bulk systems, Phys. Rev. B 85, 045116 (2012).

[74] S. Albrecht, L. Reining, G. Onida, V. Olevano, and R. Del Sole, Albrecht et al. Reply:, Phys. Rev. Lett. 83, 3971 (1999).

[75] D. Kammerlander, S. Botti, M. A. L. Marques, A. Marini, and C. Attaccalite, Speeding up the solution of the Bethe-Salpeter equation by a double-grid method and Wannier interpolation, Phys. Rev. B 86, 125203 (2012).

[76] V. Bougrov, M. Levinshtein, S. Rumyantsev, and A. Zubrilov, in Properties of Advanced Semiconductor Materials GaN, AlN, $I n N, B N, S i C, S i G e$, edited by M. Levinshtein, S. Rumyantsev, and M. Shur (Wiley, New York, 2001), pp. 1-30.

[77] M. Feneberg, M. Röppischer, C. Cobet, N. Esser, J. Schörmann, T. Schupp, D. J. As, F. Hörich, J. Bläsing, A. Krost, and R. Goldhahn, Optical properties of cubic GaN from 1 to 20 eV, Phys. Rev. B 85, 155207 (2012).

[78] M. Parenteau, C. Carlone, and S. M. Khanna, Damage coefficient associated with free exciton lifetime in GaAs irradiated with neutrons and electrons, J. Appl. Phys. 71, 3747 (1992).

[79] D. J. As, F. Schmilgus, C. Wang, B. Schöttker, D. Schikora, and K. Lischka, The near band edge photoluminescence of cubic GaN epilayers, Appl. Phys. Lett. 70, 1311 (1997).

[80] M. A. Jakobson, V. D. Kagan, R. P. Seisyan, and E. V. Goncharova, Optical properties of pure $\mathrm{CdS}$ and metalinsulator-semiconductor structures on CdS at electrical operation, J. Cryst. Growth 138, 225 (1994).

[81] J. Voigt, F. Spiegelberg, and M. Senoner, Band parameters of $\mathrm{CdS}$ and CdSe single crystals determined from optical exciton spectra, Phys. Status Solidi B 91, 189 (1979).

[82] J. F. Muth, J. H. Lee, I. K. Shmagin, R. M. Kolbas, H. C. Casey, B. P. Keller, U. K. Mishra, and S. P. DenBaars, Absorption coefficient, energy gap, exciton binding energy, and recombination lifetime of $\mathrm{GaN}$ obtained from transmission measurements, Appl. Phys. Lett. 71, 2572 (1997).

[83] P. Lautenschlager, M. Garriga, S. Logothetidis, and M. Cardona, Interband critical points of GaAs and their temperature dependence, Phys. Rev. B 35, 9174 (1987).

[84] B. Sonntag, Dielectric and optical properties, in Rare Gas Solids, edited by M. L. Klein and J. A. Venables (Academic, London, 1976), Vol. II, p. 1021.

[85] R. A. R. Leute, M. Feneberg, R. Sauer, K. Thonke, S. B. Thapa, F. Scholz, Y. Taniyasu, and M. Kasu, Photoluminescence of highly excited AlN: Biexcitons and exciton-exciton scattering, Appl. Phys. Lett. 95, 031903 (2009). 
[86] R. Haensel, G. Keitel, E. E. Koch, M. Skibowski, and P. Schreiber, Reflection Spectrum of Solid Argon in the Vacuum Ultraviolet, Phys. Rev. Lett. 23, 1160 (1969).

[87] J. A. Van Vechten and R. M. Martin, Calculation of Local Effective Fields: Optical Spectrum of Diamond, Phys. Rev. Lett. 28, 446 (1972).

[88] D. M. Roessler and W. C. Walker, Optical constants of magnesium oxide and lithium fluoride in the far ultraviolet, J. Opt. Soc. Am. 57, 835 (1967).

[89] A. Marini, R. Del Sole, and A. Rubio, Bound Excitons in Time-Dependent Density-Functional Theory: Optical and Energy-Loss Spectra, Phys. Rev. Lett. 91, 256402 (2003).

[90] J. P. Perdew, K. Burke, and M. Ernzerhof, Generalized Gradient Approximation Made Simple, Phys. Rev. Lett. 77, 3865 (1996); 78, 1396(E) (1997).

[91] I. E. Castelli, J. M. García-Lastra, K. S. Thygesen, and K. W. Jacobsen, Bandgap calculations and trends of organometal halide perovskites, APL Mater. 2, 081514 (2014).

[92] M. C. Brennan, J. E. Herr, T. S. Nguyen-Beck, J. Zinna, S. Draguta, S. Rouvimov, J. Parkhill, and M. Kuno, Origin of the size-dependent Stokes shift in $\mathrm{CsPbBr}_{3}$ perovskite nanocrystals, J. Am. Chem. Soc. 139, 12201 (2017).

[93] T. J. Whitcher, J.-X. Zhu, X. Chi, H. Hu, D. Zhao, T. C. Asmara, X. Yu, M. B. H. Breese, A. H. Castro Neto, Y. M. Lam, A. T. S. Wee, E. E. M. Chia, and A. Rusydi, Importance of Electronic Correlations and Unusual Excitonic Effects in Formamidinium Lead Halide Perovskites, Phys. Rev. X 8, 021034 (2018).

[94] L. Lang, J.-H. Yang, H.-R. Liu, H. Xiang, and X. Gong, First-principles study on the electronic and optical properties of cubic $\mathrm{ABX}_{3}$ halide perovskites, Phys. Lett. A 378, 290 (2014).

[95] L. Protesescu, S. Yakunin, M. I. Bodnarchuk, F. Krieg, R. Caputo, C. H. Hendon, R. X. Yang, A. Walsh, and M. V. Kovalenko, Nanocrystals of cesium lead halide perovskites $\left(\mathrm{CsPbBr}_{3}, \mathrm{X}=\mathrm{Cl}, \mathrm{Br}\right.$, and I): Novel optoelectronic materials showing bright emission with wide color gamut, Nano Lett. 15, 3692 (2015).

[96] J. Li, X. Yuan, P. Jing, J. Li, M. Wei, J. Hua, J. Zhao, and L. Tian, Temperature-dependent photoluminescence of inorganic perovskite nanocrystal films, RSC Adv. 6, 78311 (2016).
[97] J. Song, Q. Cui, J. Li, J. Xu, Y. Wang, L. Xu, J. Xue, Y. Dong, T. Tian, H. Sun, and H. Zeng, Ultralarge allinorganic perovskite bulk single crystal for high-performance visible-infrared dual-modal photodetectors, Adv. Opt. Mater. 5, 1700157 (2017).

[98] Z. Yang, A. Surrente, K. Galkowski, A. Miyata, O. Portugall, R. J. Sutton, A. A. Haghighirad, H. J. Snaith, D. K. Maude, P. Plochocka, and R. J. Nicholas, Impact of the halide cage on the electronic properties of fully inorganic cesium lead halide perovskites, ACS Energy Lett. 2, 1621 (2017).

[99] J. Butkus, P. Vashishtha, K. Chen, J. K. Gallaher, S. K. K. Prasad, D. Z. Metin, G. Laufersky, N. Gaston, J. E. Halpert, and J. M. Hodgkiss, The evolution of quantum confinement in $\mathrm{CsPbBr}_{3}$ perovskite nanocrystals, Chem. Mater. 29, 3644 (2017).

[100] A. Miyata, A. Mitioglu, P. Plochocka, O. Portugall, J. T.-W. Wang, S. D. Stranks, H. J. Snaith, and R. J. Nicholas, Direct measurement of the exciton binding energy and effective masses for charge carriers in organic-inorganic tri-halide perovskites, Nat. Phys. 11, 582 (2015).

[101] G. R. Yettapu, D. Talukdar, S. Sarkar, A. Swarnkar, A. Nag, P. Ghosh, and P. Mandal, Terahertz conductivity within colloidal $\mathrm{CsPbBr}_{3}$ perovskite nanocrystals: Remarkably high carrier mobilities and large diffusion lengths, Nano Lett. 16, 4838 (2016).

[102] Y. Rakita, N. Kedem, S. Gupta, A. Sadhanala, V. Kalchenko, M. L. Böhm, M. Kulbak, R. H. Friend, D. Cahen, and G. Hodes, Low-temperature solution-grown $\mathrm{CsPbBr}_{3}$ single crystals and their characterization, Cryst. Growth Des. 16, 5717 (2016).

[103] M. Bokdam, T. Sander, A. Stroppa, S. Picozzi, D. D. Sarma, C. Franchini, and G. Kresse, Role of polar phonons in the photo excited state of metal halide perovskites, Sci. Rep. 6, 28618 (2016).

[104] J. Leveillee and A. Schleife, Free-electron effects on the optical absorption of the hybrid perovskite $\mathrm{CH}_{3} \mathrm{NH}_{3} \mathrm{PbI}_{3}$ from first principles, Phys. Rev. B 100, 035205 (2019).

[105] F. A. Rasmussen, P. S. Schmidt, K. T. Winther, and K. S. Thygesen, Efficient many-body calculations for twodimensional materials using exact limits for the screened potential: Band gaps of $\mathrm{MoS}_{2}$, h-BN, and phosphorene, Phys. Rev. B 94, 155406 (2016). 